\title{
Epigenetic findings in periodontitis in UK twins: a cross-sectional study
}

\author{
Yuko Kurushima' ${ }^{1}$, Pei-Chien Tsai ${ }^{1,2,3}$, Juan Castillo-Fernandez ${ }^{1}$, Alexessander Couto Alves ${ }^{1}$, \\ Julia Sarah El-Sayed Moustafa ${ }^{1}$, Caroline Le Roy ${ }^{1}$, Tim D. Spector ${ }^{1}$, Mark Ide ${ }^{4}$, Francis J. Hughes ${ }^{4}$, Kerrin S. Small ${ }^{1}$, \\ Claire J. Steves ${ }^{1}$ and Jordana T. Bell ${ }^{1 *}$ (I)
}

\begin{abstract}
Background: Genetic and environmental risk factors contribute to periodontal disease, but the underlying susceptibility pathways are not fully understood. Epigenetic mechanisms are malleable regulators of gene function that can change in response to genetic and environmental stimuli, thereby providing a potential mechanism for mediating risk effects in periodontitis. The aim of this study is to identify epigenetic changes across tissues that are associated with periodontal disease.
\end{abstract}

Methods: Self-reported gingival bleeding and history of gum disease, or tooth mobility, were used as indicators of periodontal disease. DNA methylation profiles were generated using the Infinium HumanMethylation450 BeadChip in whole blood, buccal, and adipose tissue samples from predominantly older female twins (mean age 58) from the TwinsUK cohort. Epigenome-wide association scans (EWAS) of gingival bleeding and tooth mobility were conducted in whole blood in 528 and 492 twins, respectively. Subsequently, targeted candidate gene analysis at 28 genomic regions was carried out testing for phenotype-methylation associations in 41 (tooth mobility) and 43 (gingival bleeding) buccal, and 501 (tooth mobility) and 556 (gingival bleeding) adipose DNA samples.

Results: Epigenome-wide analyses in blood identified one CpG-site (cg21245277 in ZNF804A) associated with gingival bleeding ( $F D R=0.03$, nominal $p$ value $=7.17 \mathrm{e}-8)$ and 58 sites associated with tooth mobility $(F D R<0.05)$ with the top signals in IQCE and XKR6. Epigenetic variation at 28 candidate regions (247 CpG-sites) for chronic periodontitis showed an enrichment for association with periodontal traits, and signals in eight genes (VDR, IL6ST, TMCO6, ILIRN, CD44, ILIB, WHAMM, and CXCL1) were significant in both traits. The methylation-phenotype association signals validated in buccal samples, and a subset (25\%) also validated in adipose tissue.

Conclusions: Epigenome-wide analyses in adult female twins identified specific DNA methylation changes linked to self-reported periodontal disease. Future work will explore the environmental basis and functional impact of these results to infer potential for strategic personalized treatments and prevention of chronic periodontitis.

Keywords: Epigenetics, Periodontitis, DNA methylation, Gene expression, Metabolomics, Epigenome-wide association scan (EWAS)

\footnotetext{
* Correspondence: Jordana.Bell@kcl.ac.uk

${ }^{1}$ Department of Twin Research and Genetic Epidemiology, School of Life

Course Sciences, King's College London, London, UK

Full list of author information is available at the end of the article
}

(c) The Author(s). 2019 Open Access This article is distributed under the terms of the Creative Commons Attribution 4.0 International License (http://creativecommons.org/licenses/by/4.0/), which permits unrestricted use, distribution, and reproduction in any medium, provided you give appropriate credit to the original author(s) and the source, provide a link to the Creative Commons license, and indicate if changes were made. The Creative Commons Public Domain Dedication waiver (http://creativecommons.org/publicdomain/zero/1.0/) applies to the data made available in this article, unless otherwise stated. 


\section{Background}

Periodontal disease is a chronic inflammatory disease that poses a significant burden among older populations. The disease causes inflammation and bleeding of the gingival tissues as a result of the accumulation of microbial dental plaque, and in its destructive form, periodontitis results in progressive destruction of the supporting structures of the teeth leading to looseness of the teeth and ultimately tooth loss. The etiology of the disease involves interplay between both intrinsic and extrinsic factors such as genetic susceptibility, diet, oral hygiene, and inflammatory response to exposure to complex pathogens. An increasing number of genome-wide association studies $[1,2]$ and candidate gene reports [3-5] have explored genetic susceptibility to periodontal disease. Genes that harbor genetic variants robustly associated with the development of periodontal disease are related to inflammatory response and alveolar bone resorption; however, their mechanistic pathways are not clearly understood.

Epigenetic modifications, including DNA methylation, are key regulators of gene function. Epigenetic marks can change in response to genetic and environmental stimuli, thereby providing a mechanism for the interaction of genetic and environmental effects in the context of disease susceptibility. A number of epigenetic changes have been identified to contribute towards and act as biomarkers of human diseases, including cancers, inflammatory and neurological disorders, and mental health traits [6, 7]. However, only a few studies so far have explored epigenetics in periodontal disease, although periodontal disease is characterized by an inflammatory response. The majority of epigenetic research into periodontal disease to date has focused on candidate genes in case-control samples [8-10]. Previous studies predominantly explored the hypothesis that epigenetic regulation mediates gingival inflammation in epithelial tissue by changing the function of cytokine genes. This includes specifically the upregulation of proinflammatory cytokines and other signaling molecules and downregulation of anti-inflammatory cytokines to activate a full inflammatory response.

Although both genetic and environmental risk factors contribute to periodontal disease susceptibility, the underlying molecular pathways of these risk effects are not fully understood. Epigenetic studies may uncover mechanisms mediating genetic and environmental risk effects on periodontitis, which will help towards developing strategic personalized treatments and prevention.

The aim of the current study was to investigate epigenomic variation associated with chronic periodontal disease using a twofold approach. The first stage explored the association of periodontal traits with epigenome-wide variation in over 500 female adult twins. Thereafter, a candidate gene approach was undertaken, where we explored multi-omics signatures at candidate genomic regions previously implicated in periodontitis [1, 2, 11-13]. The association between periodontal status and DNA methylation levels was assessed in whole blood, as the most widely used sample type; in adipose tissue, as an independent sample type; and in buccal tissue, as a representative biological sample from the oral cavity.

\section{Materials and methods}

\section{Study population}

Study participants were older female twins (mean age $=$ 58 years old; age range $19-82$ years old; $83 \%$ are monozygotic (MZ) and 10\% are dizygotic (DZ)) who were enlisted in the TwinsUK registry [14]. The TwinsUK registry is one of the largest adult twin cohorts encompassing a wide range of phenotypes, and biological samples and data. Informed consent was obtained from all participants before sample and data collection. Zygosity was determined by standard questionnaire [15] and confirmed by DNA short-tandem repeat fingerprinting. The number of samples across different datasets explored in this study is described in Table 1. Gingival bleeding status was obtained from 528 female individuals (mean age 57.9, age range 18.6-80.9, mean BMI 26.9, BMI range 16.1-49.3), and tooth mobility data was obtained from 492 female individuals (mean age 58.0, age range 18.680.9, mean BMI 26.9, BMI range 16.2-52.7), for whom blood DNA methylation profiles were also available. The date of the clinical visit for periodontal data collection was within 5 years of the blood DNA extraction date. Buccal tissue DNA methylation samples included 43 female individuals (mean age 57, age range 34-73; subjects including $20 \mathrm{MZ}$ individuals, $16 \mathrm{DZ}$ individuals, and 7 singletons) with gingival bleeding phenotype and $41 \mathrm{fe}-$ males (mean age 57, range 34-73, 20 MZ individuals, 12 DZ individuals, 9 singletons) with tooth mobility phenotype. Lastly, the adipose tissue DNA methylation sample included 556 female individuals (mean age 59, range 3985, 39\% MZ) with gingival bleeding phenotype and 501 female individuals (mean age 58, range $39-85,39 \% \mathrm{MZ}$, $61 \% \mathrm{DZ}$ ) with tooth mobility phenotype.

\section{Periodontal phenotypes}

Periodontal phenotype data on tooth mobility and gingival bleeding were collected by self-reported questionnaires during clinical visits. A single question was used to determine gingival bleeding (question: "Have you ever had the condition of gum bleeding") and tooth mobility (question: "Have you ever had the condition of gum decay or loose teeth"). Participants were asked to answer either "yes" or "no" to these questions. Therefore, both phenotypes were binary variables. In the blood DNA methylation dataset, 269 individuals out of $528(51 \%)$ were gingival bleeding positive and 121 out of 492 (25\%) 
Table 1 Samples used in blood, buccal, and adipose tissue data analysis for two periodontal traits

\begin{tabular}{llll}
\hline & Gingival bleeding ${ }^{\mathrm{a}, \mathrm{b}}$ & Tooth mobility & Overlap between two periodontal traits \\
\hline Blood & & & 474 \\
DNA methylation & 528 & 492 & 333 \\
RNAseq & $342(120)$ & $335(112)$ & 454 \\
Metabolomics & $506(506)$ & $472(472)$ & 40 \\
Buccal DNA methylation & 43 & 41 & 501 \\
Adipose DNA methylation & 556 & 501 & \\
\hline
\end{tabular}

anits represent the number of individuals

${ }^{b}$ Numbers in brackets represent the number of individuals who also have DNA methylation data

were tooth mobility positive. In the buccal methylation dataset, 25 individuals out of 43 were gingival bleeding positive and 12 individuals out of 41 were tooth mobility positive. In the adipose DNA methylation dataset, 265 individuals out of 556 were gingival bleeding positive and 102 individuals out of 501 were tooth mobility positive.

\section{Epigenome-wide profiling of DNA methylation}

The Infinium HumanMethylation450 BeadChip Kit (Illumina Inc., San Diego, CA, USA) was used to obtain the ratio of array intensity signal from the methylated beads over the sum of methylated and unmethylated bead signals plus 100. This array contains a combination of two types of probes, Infinium I (28\%) and Infinium II (72\%) probes, which use different detection methods. Infinium I probes include two bead types, a methylated and an unmethylated, while Infinium II probes are measured in one bead type that requires extension of color-labeled dideoxynucleotides to determine the methylation status. To correct for technical bias caused by the probe design in this platform, the beta-mixture quantile normalization (BMIQ) method and background correction were applied for each sample [16]. Subjects with more than $1 \%$ of missing values were considered as outliers and were removed from the analysis. Probes with more than $1 \%$ of missing values among all samples with detection $P$ value $>0.05$, probes located on Y chromosome, and probes that mapped incorrectly or that mapped to multiple locations in the genome (with up to two mismatches) were removed. We also used the 65 designed single nucleotide polymorphism (SNPs) to check for sample swaps and included these probes in the analysis. In total, after all exclusions, we analyzed 452,865 probes in blood, 436,814 probes in buccal tissue, and 467,928 probes in adipose samples. The methylation beta levels at each probe were quantile normalized to the standard normal distribution $(N(0,1))$ before downstream epigenome-wide association analysis. Epigenome-wide association scans aimed to identify specific sites across the genome where epigenetic levels significantly differed between periodontal diseasepositive and periodontal disease-negative group samples, that is, identifying differentially methylated positions
(DMPs) for periodontal disease. CpG sites that surpassed epigenome-wide significance did not overlap common genetic variants (minor allele frequency $>5 \%$ ) in the UK10K project [17].

\section{RNA-sequencing gene expression quantification in whole blood}

RNA-sequencing (RNASeq) data from peripheral whole blood samples were available for 384 subjects and were generated, quantified, and normalized as previously described $[18,19]$. Briefly, RNASeq reads were aligned to the UCSC (University of California Santa Cruz) Genome Browser GRCh37 reference genome with the Burrows-Wheeler Aligner [20], and genes annotated using GENCODE v.10. Samples with fewer than 10 million reads mapping to known exons were excluded. All read count quantifications were carried out at the exon level and were corrected for variation in sequencing depth between samples by normalizing the number of reads to the median number of well-mapped reads. Exons expressed in fewer than $90 \%$ of individuals were excluded. Rank-based inverse normal transformation was then applied to the normalized read counts prior to downstream analysis. The number of individuals included in the gene expression analysis with available gingival bleeding and with tooth mobility phenotypes was 342 and 335, respectively.

\section{Metabolomics data}

Fasting whole blood samples were extracted and stored at $-80^{\circ} \mathrm{C}$ until they were processed by Metabolon, Inc. (Durham, NC, USA) using a non-targeted mass spectrometry platform, as previously described [21]. After profiling, normalization was performed to correct for variation resulting from instrument inter-day tuning differences. Briefly, each compound was normalized so that the median of the run-day was equal to one (1.00) and each data point was scaled proportionally. The analysis only focused on 271 known metabolites, which included amino acids, carbohydrates, vitamins, lipids, nucleotides, small peptides, and xenobiotics. This metabolomics data was available in 472 (tooth mobility) and 506 (gingival bleeding) individuals for whom phenotype and whole 
blood DNA methylation data were also profiled. The overlap in datasets across the two periodontal traits, DNA methylation, and blood gene expression and metabolomics samples is shown in Table 1 .

\section{Candidate gene selection}

We focused on 37 candidate genes for periodontitis (Table 2). Some of these genes were selected as they were previously identified through genome-wide association studies (GWAS) of periodontitis, while other regions were included because of their biological function and previous research exploring their contribution to periodontal disease (see Table 2). Each candidate genomic region was defined by the following criteria: for GWAS SNPs, the region was set with a range of $10 \mathrm{~kb}$ around the lead SNP, and for biological candidate genes, we included the gene body and $5 \mathrm{~kb}$ either side of the gene boundary. In total, 28 of the 37 candidate genes were represented on the Infinium HumanMethylation450 array and 247 CpG sites passed quality control assessment in blood.

Further to the epigenome-wide analyses in whole blood, we assessed the association between periodontal traits and buccal tissue DNA methylation at the candidate CpG sites, as well as that for adipose tissue DNA methylation. Buccal DNA methylation analyses were carried out in 41 (tooth mobility) and 43 (gingival bleeding) individuals at a subset of $248 \mathrm{CpG}$ sites that passed quality control assessment in the buccal dataset.

\section{Statistical analysis}

We performed a linear mixed-effect regression (LMER) model to assess the association between DNA methylation levels at each probe and periodontal status of the individual. We initially tested a full linear regression model where normalized DNA methylation levels were the response variable and each periodontal phenotype was a fixed effects predictor, along with a number of covariates. Covariates included fixed effects (age, Illumina beadchip, Illumina beadchip position, BMI, smoking status, blood cell composition (monocytes, natural killer cells, granulocytes, CD8 T cells, and CD4 T cells), DNA extraction year, and time difference between the time point of dental phenotype collection and DNA extraction) and random effects (family structure and zygosity) variables. Blood cell counts were estimated using the DNA Methylation Age Calculator online [22]. The fit of the full linear regression model was compared to the fit of a null model, which excluded the dental phenotype as a predictor variable. The association between DNA methylation levels and periodontal traits were carried out using lmer within R 3.4.3 in the package "Ime4." In buccal samples, the association between periodontal status and methylation levels was also assessed using LMER adjusting for fixed effects (age and beadchip) and random effects (zygosity and family structure) as in blood. In adipose samples fixed effects included age, beadchip, BMI, bisulfite conversion level, and bisulfite efficiency, and random effects were as described in blood. Multiple testing correction was carried out using a false discovery rate (FDR) at 5\% [23]. Similarly, we tested if exon expression levels were differentially associated with periodontal traits using a linear mixed-effects regression model. The analysis was conducted for each exon adjusting for fixed effects (age, age squared, smoking and GC mean, insert size mode) and random effects (family structure, zygosity, date, and primer index).

Heritability estimation was conducted using a classical twin study model. The phenotypic variance is partitioned into three latent components: additive genetic, shared environmental, and unique environmental factors. In this context, shared environmental factors typically represent familial exposures shared by both twins (for example, in childhood), whereas unique environmental factors are exposures that are specific to each individual subject. Structural equation modeling was used to estimate the best-fitting model for the traits using the likelihood ratio test [24]. The contribution of each latent component to the phenotypic variance was also quantified. We calculated heritability in $\mathrm{R}$ with the "mets" package.

\section{Results}

ZNF804A methylation associates with gingival bleeding Epigenome-wide analysis of gingival bleeding was carried out in the blood methylome across 452,865 probes in 528 subjects (Table 1 ). We identified one CpG site significantly associated with gingival bleeding (cg21245277; beta $=-0.33, p$ value $=7.17 \mathrm{e}-8, \mathrm{FDR}=0.03$; Table 3 , Additional file 1: Table S1). This CpG site is located within the 5' UTR of a protein-coding gene, ZNF804A (Fig. 1a). Polymorphisms of this gene confer increased susceptibility to mental health disorders such as schizophrenia and bipolar disorders and contribute to heroin addiction [25]. CpG site cg21245277 was significantly hypo-methylated in participants who experienced gingival bleeding (Fig. 1b).

To assess if genetic or environmental factors influence blood DNA methylation levels at this site, we carried out heritability analysis using the classical twin model. We established a moderate impact of genetic variation on DNA methylation variability at this CpG site (heritability of $23 \%$ ), while the majority (77\%) of methylation variance was attributed to environmental factors unique to each individual.

To explore the potential functional role of the methylation signal in ZNF804A, we carried out additional omic analyses. First, RNA-sequencing gene expression data in 342 twins identified two exons in ZNF804A, which are expressed in blood. One exon showed a nominally 
Table 2 Chronic periodontitis GWAS loci and candidate genes from previous studies

\begin{tabular}{|c|c|c|c|c|}
\hline Gene symbol ${ }^{a}$ & $450 \mathrm{~K}$ probes $^{\mathrm{b}}$ & SNPS & Functional association with periodontitis or other diseases & References \\
\hline NIN & 2 & rs12883458 & \multirow[t]{3}{*}{ Genome-wide significant association with severe chronic periodontitis } & \multirow{5}{*}{$\begin{array}{l}\text { Rhodin et al. } \\
\text { [1] }\end{array}$} \\
\hline$A B H D 12 B$ & 2 & rs12883458 & & \\
\hline WHAMM & 9 & rs2890313 & & \\
\hline KCNK1 & 0 & rs11800854 & Genome-wide significant association with red complex high colonization & \\
\hline DAB2IP & 0 & rs10760187 & Genome-wide significant association with Porphyromonas gingivalis high colonization & \\
\hline CLEC19A & 1 & rs1156327 & \multirow{6}{*}{$\begin{array}{l}\text { Genome-wide significant association with PCT1 (microbial community structure that has } \\
\text { a high correlation and positive loading with all pathogens) }\end{array}$} & \multirow{12}{*}{$\begin{array}{l}\text { Offenbacher } \\
\text { et al. [2] }\end{array}$} \\
\hline TRA & 0 & rs3811273 & & \\
\hline TM9SF2P & 1 & rs9557237 & & \\
\hline GGTA2P & 0 & rs17184007 & & \\
\hline$|F| 16$ & 1 & rs1633266 & & \\
\hline RBMS3 & 0 & rs17718700 & & \\
\hline C1QTNF7 & 0 & rs4074082 & \multirow{2}{*}{$\begin{array}{l}\text { Genome-wide significant association with PCT3 (high positive loading of GCF-IL-1 } \beta \text { and } \\
\text { Aggregatibacter actinomycetemcomitans) }\end{array}$} & \\
\hline TSNARE & 1 & rs9772881 & & \\
\hline HPVC1 & 0 & rs10232172 & $\begin{array}{l}\text { Genome-wide significant association with PCT4 (mixed-infection community with high } \\
\text { positive loading of GCF-IL1, weak positive loadings of } P n, P i \text {, and } T d \text {, and low levels of } A a \text { ) }\end{array}$ & \\
\hline SLC15A4 & 1 & rs7135417 & \multirow[t]{3}{*}{ Genome-wide significant association with PCT5 (chronic periodontitis) } & \\
\hline PKP2 & 2 & rs6488099 & & \\
\hline SNRPN & 9 & rs904310 & & \\
\hline $1 / 8$ & 3 & & $\begin{array}{l}\text { Regulation of innate and acquired immune response } \\
\text { Increased in gingival crevicular fluid from patients with periodontitis }\end{array}$ & \multirow[t]{11}{*}{$\begin{array}{l}\text { Zhan et al. } \\
\text { [11] }\end{array}$} \\
\hline CD44 & 34 & & Smoking-induced periodontal tissue destruction & \\
\hline CXCL1 & 7 & & $\begin{array}{l}\text { Stimulation of lipopolysaccharide in oral fibroblasts which leads to development of } \\
\text { periodontal inflammation }\end{array}$ & \\
\hline IL6ST & 17 & & $\begin{array}{l}\text { Signal transducer for the interleukin family; regulates osteoclasts and calcium } \\
\text { homeostasis }\end{array}$ & \\
\hline CCR1 & 11 & & Trigger of bone resorption process & \\
\hline MMP7 & 7 & & Innate host defense in periodontal tissues & \\
\hline MMP13 & 10 & & Immunoreactivity in gingival crevicular fluid & \\
\hline MMP3 & 12 & & Physiological tissue remodeling of periodontal tissue & \\
\hline TLR9 & 0 & & Protective role against alveolar bone loss in the absence of Porphyromonas gingivalis & \\
\hline IL18 & 15 & & $\begin{array}{l}\text { Inflammation and modulation of the activity of macrophages and neutrophils. } \\
\text { Significantly higher in patients with chronic periodontitis compared to healthy controls }\end{array}$ & \\
\hline IFNB1 & 0 & & $\begin{array}{l}\text { TNFa, via the autocrine action of IFNbeta, differentially regulates the expression of } \\
\text { proinflammatory genes and DNA synthesis }\end{array}$ & \\
\hline GLT6D1 & 2 & rs1537415 & Genome-wide significant association with aggressive periodontitis & $\begin{array}{l}\text { Schaefer et } \\
\text { al. [12] }\end{array}$ \\
\hline$I L 1 B$ & 8 & & \multirow{8}{*}{$\begin{array}{l}\text { The carriage rate of the } R \text { allele (frequency of } N / R \text { and } R / R \text { genotypes) among cases and } \\
\text { controls }\end{array}$} & \multirow{8}{*}{$\begin{array}{l}\text { Laine et al. } \\
\text { [13] }\end{array}$} \\
\hline ILIRN & 13 & & & \\
\hline IL6 & 14 & & & \\
\hline IL10 & 7 & & & \\
\hline$V D R$ & 23 & & & \\
\hline CD14 & 29 & & & \\
\hline TLR4 & 4 & & & \\
\hline MMP1 & 11 & & & \\
\hline
\end{tabular}


Table 3 The most associated epigenome-wide significant signals for both periodontal traits

\begin{tabular}{|c|c|c|c|c|c|c|c|}
\hline & $b p^{a}$ & Annotation & Estimate $^{b}$ & $P$ value & FDR & Gene $^{c}$ & Reported traits \\
\hline Gingival bleeding & CHR2: 185463218 & 1st exon; 5' UTR & -0.33 & $7.17 \mathrm{e}-8$ & 0.03 & $\begin{array}{l}\text { ZNF804A } \\
\text { (Zinc Finger Protein 8) }\end{array}$ & $\begin{array}{l}\text { Bipolar disorder } \\
\text { Schizophrenia } \\
\text { Memory loss }\end{array}$ \\
\hline \multirow[t]{2}{*}{ Tooth mobility } & $\begin{array}{l}\text { CHR7: } \\
2646478\end{array}$ & Body & 0.38 & $6.85 e-8$ & $2.5 \mathrm{e}-4$ & $\begin{array}{l}\text { IQCE } \\
\text { (IQ Motif Containing E) }\end{array}$ & $\begin{array}{l}\text { Longevity } \\
\text { Blood metabolite level }\end{array}$ \\
\hline & CHR8: 11058145 & Body & -0.49 & $1.53 \mathrm{e}-8$ & $2.8 e-3$ & $\begin{array}{l}\text { XKR6 } \\
\text { (XK Related 6) }\end{array}$ & $\begin{array}{l}\text { Asthma } \\
\text { Allergic rhinitis }\end{array}$ \\
\hline
\end{tabular}

${ }^{a}$ Coordinate based on hg19

${ }^{b}$ Estimate represents the coefficient generated from the epigenetic linear regression model

'Gene name derived from UCSC

significant association with the presence of gingival bleeding (beta $=2.17 \mathrm{e}-6, p$ value $=0.018$; Fig. $1 \mathrm{c}$ ) in a model adjusting for age, smoking status, family structure, and technical covariates. Subjects who experienced gingival bleeding had lower levels of DNA methylation in the $5^{\prime}$ UTR of this gene and higher levels of exon expression compared to controls, suggesting negative epigenetic regulatory effects of cg21245277 on exon expression (correlation $=-0.15, p$ value $=0.11$ ).

To further explore the functional relevance of the most associated gingival bleeding DNA methylation signal in ZNF804A, we correlated the methylation levels at the most associated CpG site in this gene (cg21245277) with 271 known fasting blood metabolites profiled by mass spectrometry [21]. Eight metabolites were found to be nominally associated with ZNF804A cg21245277 blood DNA methylation levels (Fig. 1d). The strongest signal was observed for ornithine ( $p$ value $=0.008$ ), which is also significantly associated with tooth mobility $(p$ value $=0.012$ ) The remaining associated metabolites also included 3 -dehydrocarnitine, ranked fifth ( $p$ value $=0.029)$, which is consumed by gram-negative and gram-positive bacteria in either aerobic or anaerobic environmental insults or as a sole carbon, nitrogen, and energy source [26].

\section{Epigenome-wide analyses of self-reported periodontitis}

Epigenome-wide analyses of self-reported periodontitis (tooth mobility) identified 58 epigenome-wide significant CpG sites (Fig. 2a), although the results showed evidence for moderate genomic inflation (lambda $=1.27$ ). The two most associated CpG sites (Table 3) were located in the gene body of the IQCE gene $(\mathrm{cg} 08157914$; beta $=0.38, p$ value $=6.85 \mathrm{e}$ -8 , FDR $<0.001$, Additional file 2: Table S2) and in the gene body of the XKR6 gene (cg11051055; beta $=-0.49, p$ value $=1.53 \mathrm{e}-8, \mathrm{FDR}=0.003$, Additional file 3: Table S3).

Three (cg18353661, cg23681110, cg08453750) out of $58 \mathrm{CpG}$ sites were located in or near to the genes that also showed exon expression levels with a nominally significant association with tooth mobility (Fig. 2b). Twin-based heritability estimates decomposing the impact of genetic, shared environmental, and unique environmental effects on the variance of each blood DNA methylation level showed few strongly heritable sites (heritability $>20 \%$ at 12 CpG sites), while the majority of signals were explained by environmental contributions (Fig. 2b). Interestingly, a common environmental component was identified at 12 out of the $58 \mathrm{CpG}$ sites.

We explored if the inclusion of technical and biological covariates could attenuate the genomic inflation observed in the EWAS of tooth mobility. When we re-analyzed the data using additional participants, that is, including subjects who had dental visits more than 5 years before and after the DNA extraction time $(n=717)$, the genomic inflation factor was attenuated (lambda $=1.14)$ and only one epigenome-wide significant signal remained. This signal was the top-ranked $\mathrm{CpG}$ site from the set of $58 \mathrm{CpG}$ reported above at cg08157914 in $I Q C E$ (beta $=0.35, p$ value $=1.02-8, \mathrm{FDR}=0.007)$.

\section{Enrichment of differential methylation at chronic periodontitis GWAS loci and biological candidates across tissues}

We integrated our genome-wide differential methylation profiles in periodontal disease with previously published candidate regions for chronic periodontitis. Altogether, we explored data at 28 regions (247 CpG sites) that were previously identified either through GWAS efforts or biological studies into periodontal disease (Table 2). We observed evidence for enrichment of epigenetic associations with both periodontal traits in the blood epigenome-wide results (Fig. 3a). In blood, $26 \mathrm{CpG}$ sites (tooth mobility) and 15 CpG sites (gingival bleeding) were nominally significantly associated with dental phenotypes. CpG sites that were nominally significantly associated with both periodontal traits mapped to eight genes (VDR, IL6ST, TMCO6, IL1RN, CD44, IL1B, WHAMM, and CXCL1).

To assess the persistence of dental phenotype associations across tissues, we carried out additional epigenetic analyses between both periodontal traits and two further epigenetic datasets from buccal and adipose tissue. In buccal tissue, a stronger phenotype association enrichment was observed compared to that in blood (Fig. 3b), and 51 (tooth mobility) and 38 (gingival bleeding) CpG sites were nominally associated with the dental phenotypes. CpG sites that were nominally significantly 


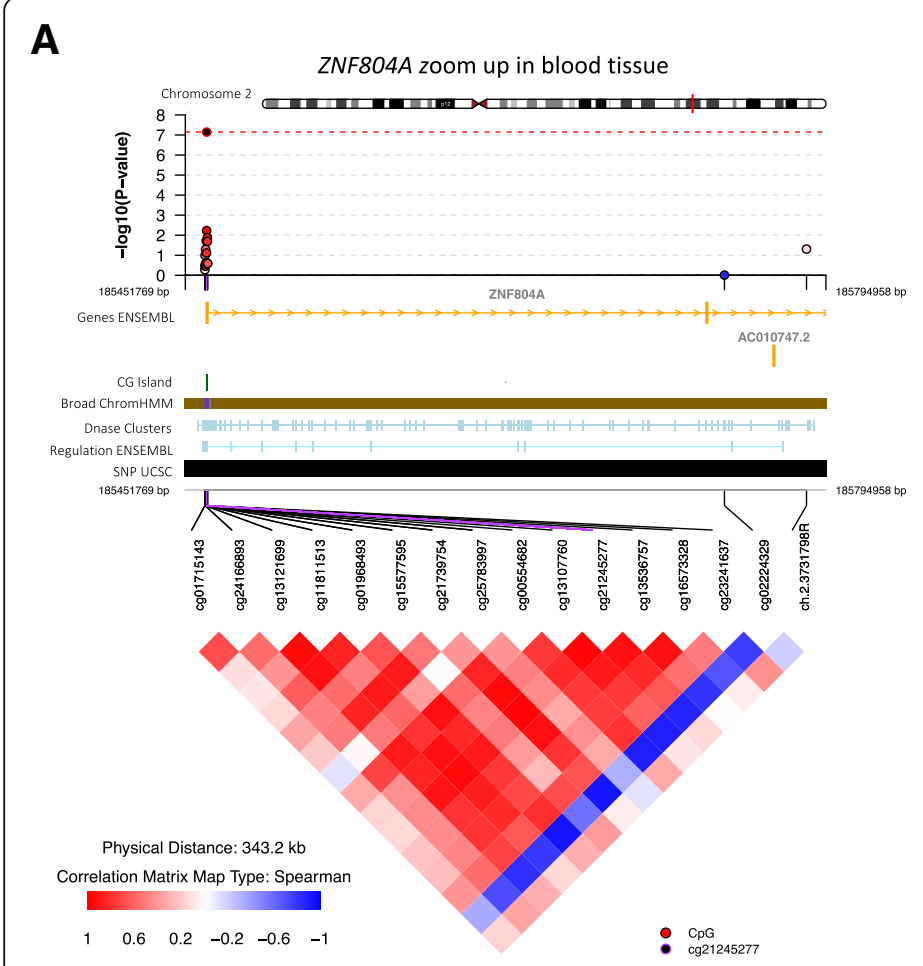

B

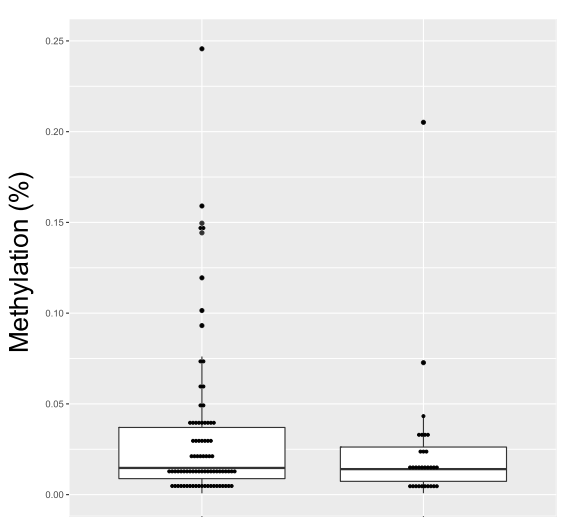

C

Gum bleeding (-) Gum bleeding (+)

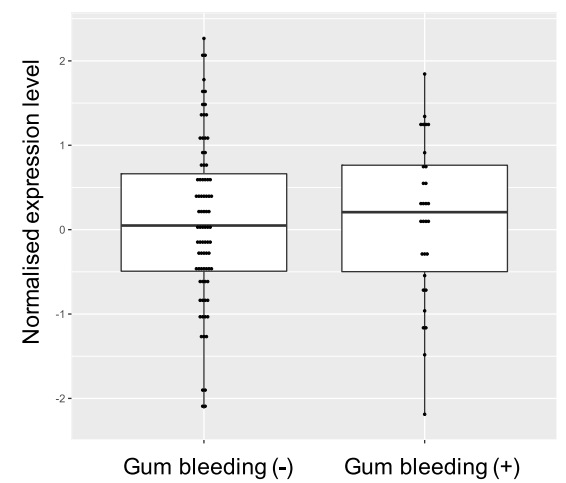

D

Blood metabolites in association with ZNF804A methylation

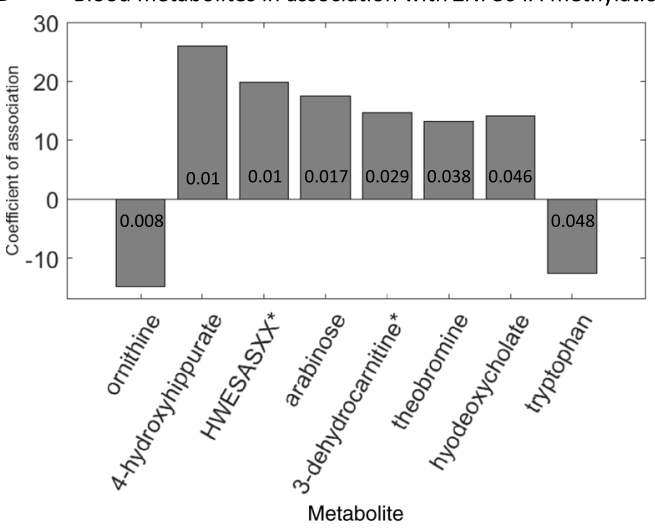

Fig. 1 ZNF804A differentially methylated signal for gingival bleeding in whole blood. a Visualization of the peak blood epigenome-wide signal for gingival bleeding (top), along with genomic annotation of the region (middle) and co-methylation patterns (bottom panel). b The association between ZNF804A blood methylation at cg21245277 and gingival bleeding. The y axis shows uncorrected methylation levels. c The association between the normalized expression levels at exon ENSG00000170396.6_185463093_185463797 in ZNF804A and gingival bleeding ( $p$ value = 0.018). $\mathbf{d}$ The association between ZNF804A blood methylation levels at cg21245277 and eight metabolites. The $y$-axis represents the coefficient of the model, and the $p$ value of the association is shown in each bar

associated with both periodontal traits mapped to 16 genes (IL1RN, IL1B, CD44, VDR, MMP13, MED24, CCR1, TMCO6, MMP3, IL6ST, TLR4, IL6, IL10, CXCL1, WHAMM, and SNORD124), which included all 8 genes detected in blood. In summary, enrichment of dental phenotype association $p$ values was observed in the blood and buccal epigenetic profiles, while in contrast, no enrichment was observed in adipose tissue (Fig. 3c).
We further explored the eight genes at which nominally significant differential methylation was detected in both blood and buccal tissue across both tooth mobility and gingival bleeding phenotypes. First, we carried out tests of association between the phenotype-associated blood DNA methylation signals and gene expression levels at these eight genes within the linear mixed effects regression framework. We found Bonferroni significant 


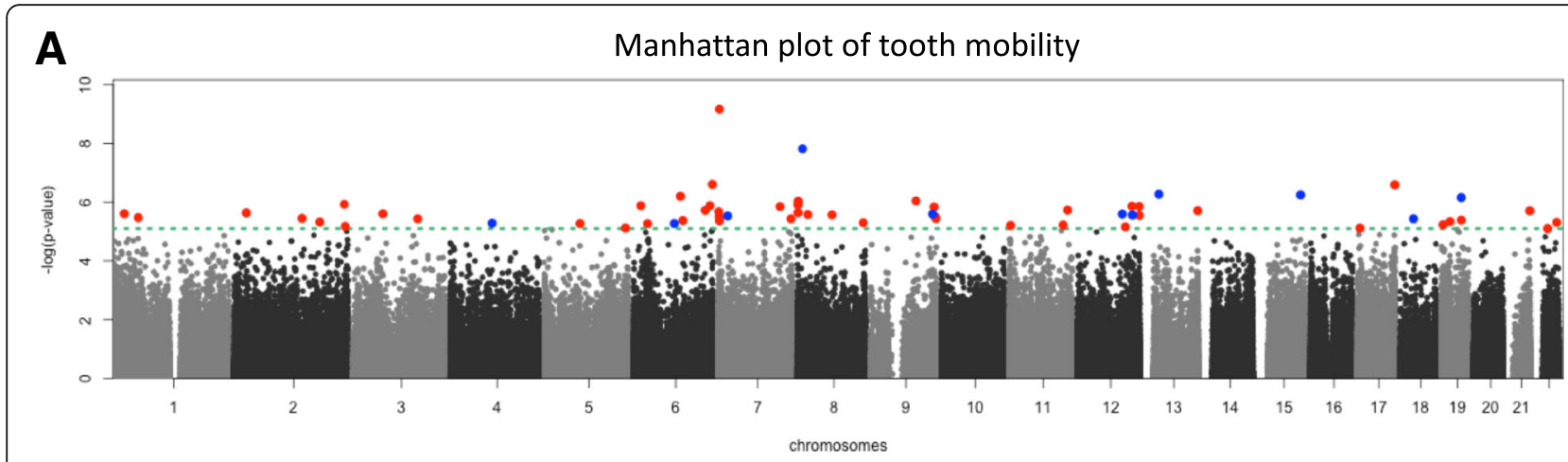

\section{B Heritability of the methylation variance at $58 \mathrm{CpGs}$}

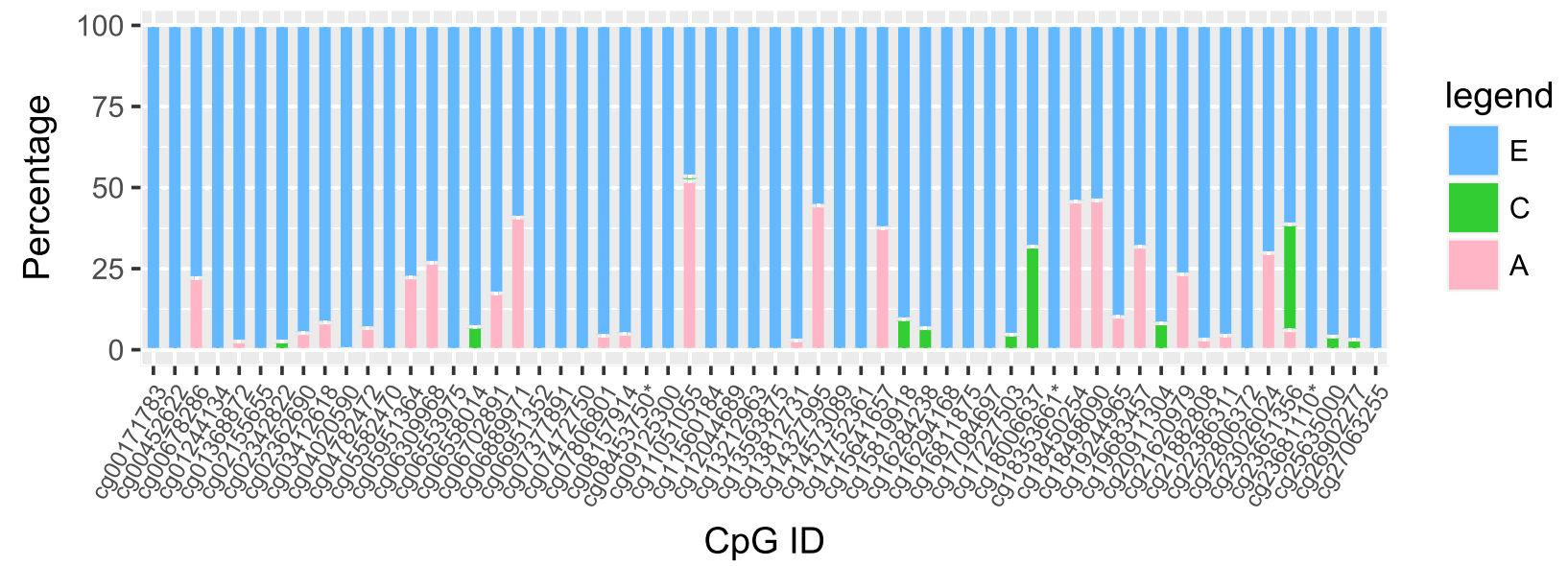

Fig. 2 Epigenome-wide association results on tooth mobility. a Epigenome-wide association results, where the green dotted line shows the epigenome-wide significance threshold (8.00e-06). There are 58 epigenome-wide significant associations with tooth mobility, showing positive (red) and negative (blue) correlation with tooth mobility. $\mathbf{b}$ The percentage of each genetic, shared environmental, and unique environmental factor contribution to the variance in methylation levels at the $58 \mathrm{CpG}$ sites. Pink (A, heritability), green (C, common environment), and blue ( $\mathrm{E}$, unique environment) bars represent genetic, shared environmental, and unique environmental effects, respectively. Three CpGs denoted by "asterisk" are located in or near to genes that also showed exon expression levels with a nominally significant association with tooth mobility

associations $(p=0.00625)$ between periodontal traits and exon expression levels for exons in WHAMM (exon ENSG00000156232.5_83499351_83499831: beta $(\mathrm{SE})=2.42 \mathrm{e}-6 \quad(7.83 \mathrm{e}-7) ; \quad p \quad$ value $=2.2 \mathrm{e}-3, \quad$ exon ENSG00000156232.5_83495140_83495248: beta $(\mathrm{SE})=$ $2.68 \mathrm{e}-6 \quad(9.13 \mathrm{e}-7) ; \quad p \quad$ value $=3.6 \mathrm{e}-3, \quad$ exon ENSG00000156232.5_83477973_83479087: beta $($ SE) $=$ $2.73 \mathrm{e}-6$ (9.36e-7); $p$ value $=3.9 \mathrm{e}-3$ ) and TMCO6 (exon ENSG00000113119.8_140023365_140023551: beta (SE) $=-0.37(0.13) ; p$ value $=6.0 \mathrm{e}-3)$. Second, to further explore their functional contribution, we examined the association between the methylation levels of the phenotype-associated $\mathrm{CpG}$ sites in these two genes (cg00115297 in WHAMM and cg00916199 in TMCO6) and fasting blood metabolites profiles. Nominally significant associations with methylation levels at these CpGs were observed for 48 metabolites (Fig. 4), with the most associated signals for lipid-related metabolites. Six out of 23 identified metabolites in a nominally significant association with WHAMM methylation were related to phosphocholine metabolism and the other three were sulfate metabolites. With respect to the association with TMCO6 methylation, 8 out of 25 identified metabolites were related to carnitine.

\section{Discussion}

We present the first large-scale epigenome-wide study of periodontitis. Our results identified differential methylation at specific CpG sites between periodontal trait positive and negative individuals, which suggests that epigenetic changes may help explain the variation in the susceptibility to chronic periodontitis. Our findings detected hypo-methylation in the 5' UTR of ZNF804A and corresponding upregulation of gene expression in individuals who experience gingival bleeding. These data suggest that ZNF804A should be considered as a candidate gene related to gingival inflammation. Blood metabolomics profile analysis highlighted eight metabolites, 

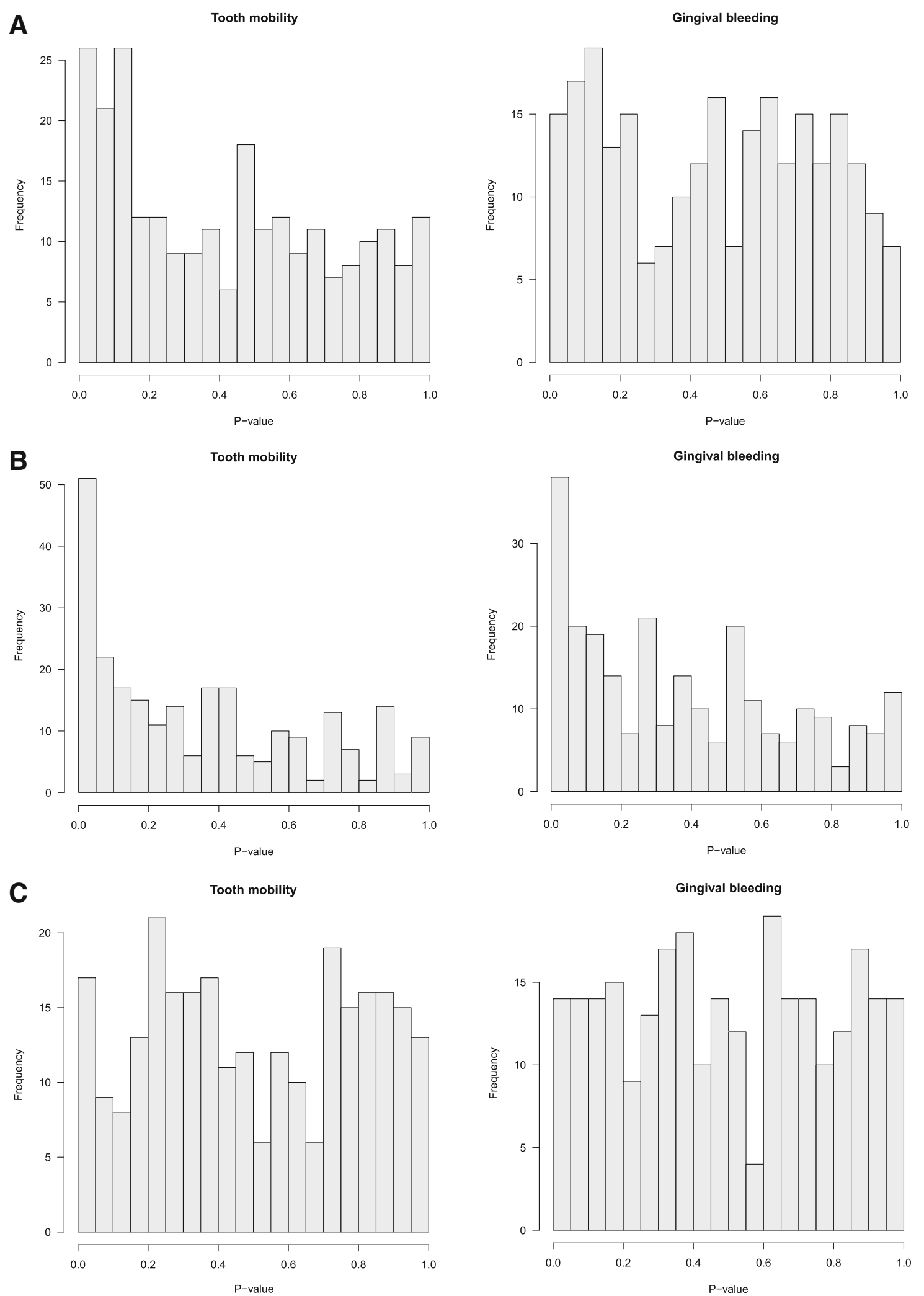

Fig. 3 Evidence of methylation-phenotype association at candidate regions for chronic periodontitis across three tissues. The histograms show the strength of association between methylation levels in blood (a), buccal (b), and adipose (c) tissues at CpG sites mapping to 28 candidate regions for periodontal traits 


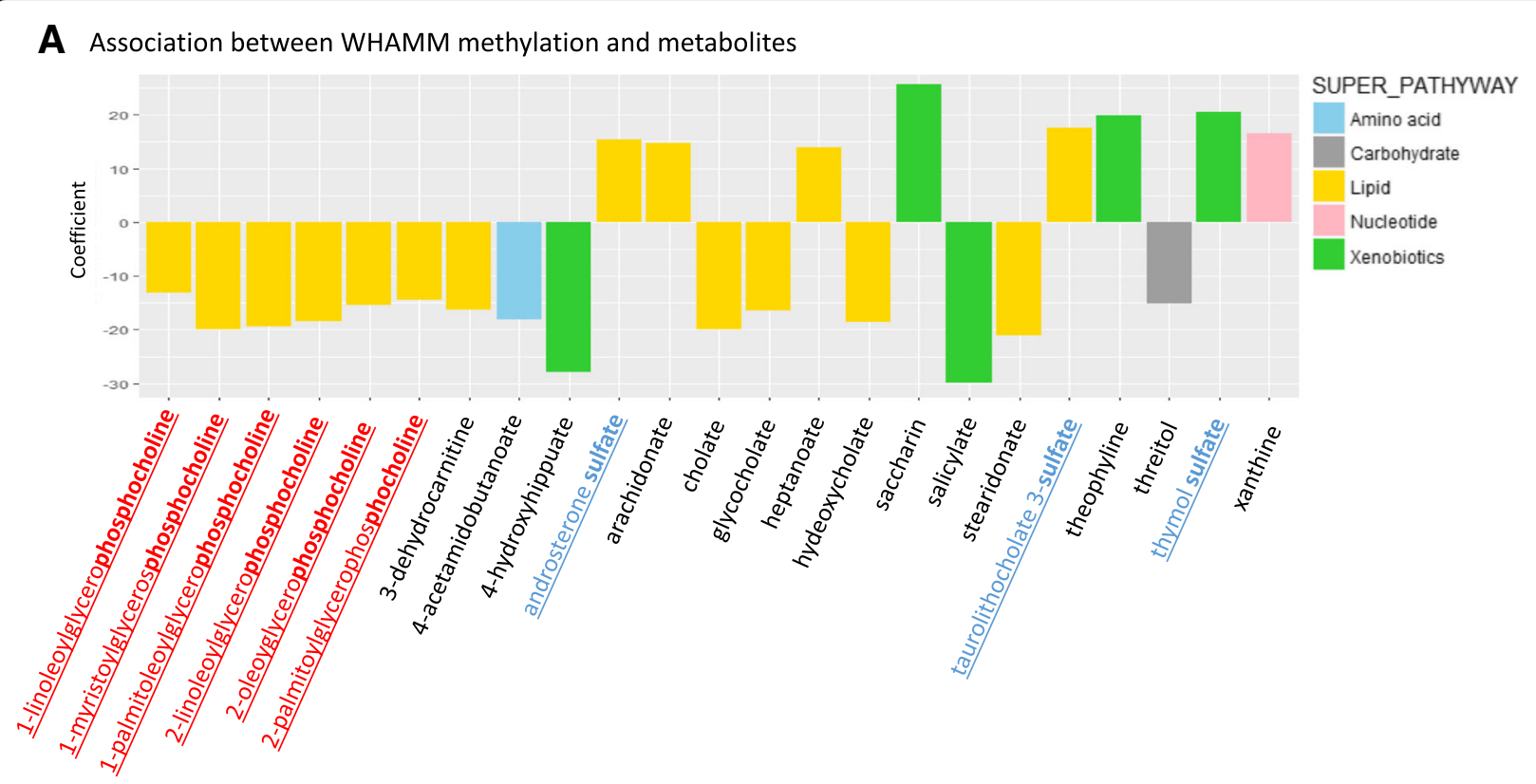

\section{B Association between TMCO6 methylation and metabolites}

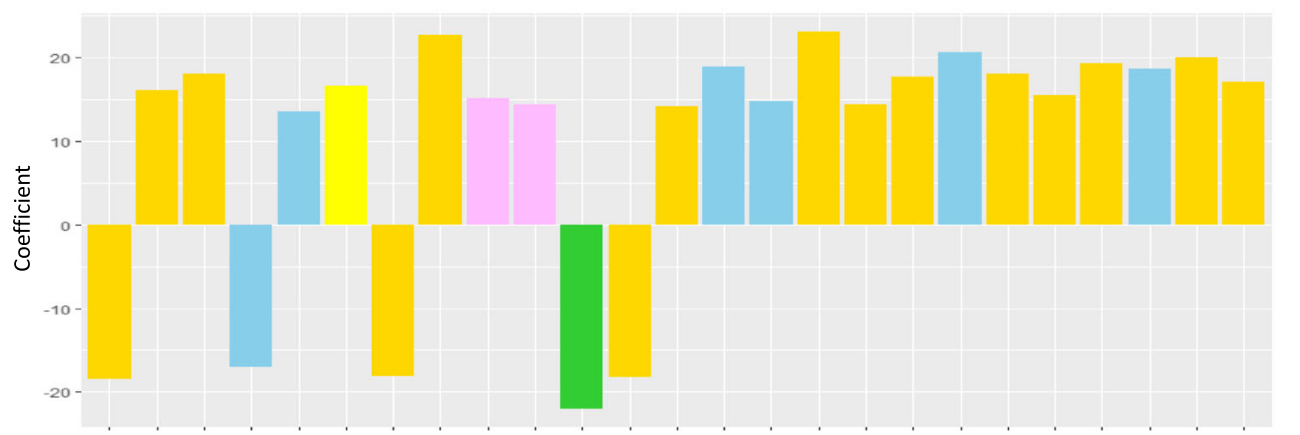

SUPER_PATHYWAY

Amino acid

Cofactors and vitamins

Lipid

Peptide

Xenobiotics

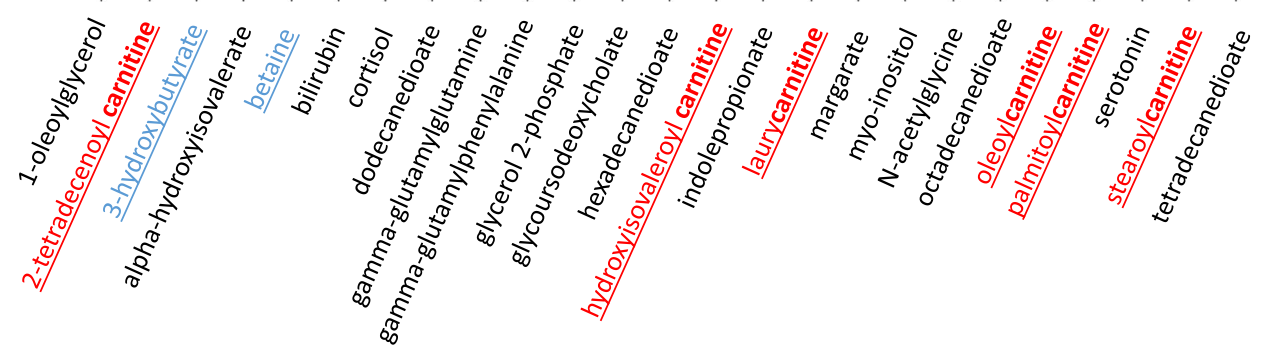

Fig. 4 Functional metabolomic characterization of WHAMM and TMCO6 methylation signals. Blood metabolites showed a nominally significant association with blood methylation levels at cg00115297 proximal to WHAMM (a) and at cg00916199 proximal to TMCO6 (b). The $y$-axis shows the coefficient of the association between methylation and metabolites level

including ornithine and carnitine, which were significantly associated with the differential methylation signal in ZNF804A. Ornithine was reported to play an important role on metabolic cross-feeding between Streptococcus gordonii and Fusobacterium nucleatum [27]. S. godonii is thought to express an arginine-ornithine antiporter, which potentially could influence the interbacterial communication with periodontal pathogens.
Kuboniwa's group also reported that the level of ornithine in saliva showed a decreasing trend after an intervention of debridement [28]. Taken together, these findings suggest a potential sequence of events in early stage periodontal disease that may lead towards disease progression, where in early stage periodontal diseasewhere gingival inflammation is observed-hypomethylation of cg21245277 may upregulate the expression of 
ZNF804A, which in turn could elevate the level of ornithine in blood.

In terms of more severe stages of periodontal disease, where tooth mobility or self-reported history of gum disease is a more appropriate phenotype marker, we identified $58 \mathrm{CpG}$ sites with epigenome-wide significant signals, although the observed genomic inflation may result in an increase in the number of false positive findings that we report here [29]. The most strongly associated signal for this phenotype was in the gene body of the IQCE gene and was hyper-methylated in individuals who presented with tooth mobility, the severe stage of periodontitis. However, there was not a significant signal for differential gene expression in this gene. Gene expression patterns associated with methylation levels at tooth mobility-associated signals were obtained at three $\mathrm{CpG}$ sites (out of $58 \mathrm{CpG}$ sites) and in three genes (MAD1L1, TRAPPC9, and OSBPL5). Epigenetic regulation may impact gene expression levels at these genes in severe cases of periodontal disease. According to the heritability findings for the variance of blood DNA methylation levels, $21 \%$ of the $58 \mathrm{CpG}$ sites showed more than $20 \%$ genetic influence, and similarly, $23 \%$ of the variance of the methylation level on the CpG site related to ZNF804A gene was explained by a genetic component. This finding is consistent with previous reports that DNA methylation levels can be under genetic control [30].

The most striking findings in our study were generated from a candidate gene approach. We identified eight genes (VDR, IL6ST, TMCO6, IL1RN, CD44, IL1B, WHAMM, and CXCL1) that displayed methylation associations with both periodontal traits across blood and buccal tissues. Of these, WHAMM and TMCO6 were identified as likely signals of genomic regulation in periodontal disease, due to a significant association between phenotype-associated methylation and gene expression levels at these genes. Both findings suggest that WHAMM and TMCO6 are strong candidates to shed light on epigenetic modifications over the course of periodontal disease. WHAMM encodes a protein which regulates the membrane dynamics and functions at the interface of microtubule and actin cytoskeletons. This gene was previously identified as a candidate from two different GWAS studies in chronic periodontitis [1,31]. TMCO6 is located near $C D 14$, which is an important contributor to periodontal inflammation as it encodes a protein which is part of the innate immune system and also recognizes lipopolysaccharides (LPS) components of gram-negative bacterial cell wall. Several case-control studies also found increased serum concentration of CD14 among the patients with periodontitis [32, 33].

Further analysis with blood metabolites showed that the epigenetic modifications at both WHAMM and TMCO6 were related to lipid metabolite biological pathways. Lipid metabolism has been well studied in microbial organisms including in periodontal pathogenic bacteria such as
Porphyromonas gingivalis and Aggregatibacter actinomycemcomitans [34-36]. Approximately 30\% of the metabolites associated with TMCO6 methylation were related to carnitine metabolism, which can be utilized by both Gram-negative and Gram-positive bacteria [37]. Three out of 23 identified metabolites related to WHAMM methylation levels were sulfates. Sulfate is known as a crucial biological marker to evaluate periodontal disease as it is one of the chemical components of glycosaminoglycans [38], which are generally elevated under inflammatory condition.

Our study has a number of limitations. Firstly, we used self-reported data to assess periodontal disease status. This is likely to underestimate the presence of disease compared to the observational data including periodontal pocket depth, attachment loss, and bleeding on probing in person by dental professionals. Our previous validation study of the self-reported data, which included 80 individuals, showed that self-reported history of gum disease or tooth mobility had low sensitivity, 0.28 , but a very high specificity and positive predictive value of 1 [39]. This result suggests that there is a high degree of certainty about the periodontitis phenotype in test subjects when compared against a control group of unknown periodontal phenotype, which arguably decreases the power of the study to detect a true difference between groups, but makes a type I error unlikely. This is consistent with previous studies of self-reported periodontitis where questions assessing loose teeth and professional diagnosis of gum disease are good predictors of periodontitis compared to other questions, but again, as with our study, with low sensitivity [40, 41]. For gingival bleeding, there was sensitivity of 0.48 and specificity of 0.75 , respectively. There is very little existing data that demonstrate the reliability of self-reported gingival bleeding, and thus, our results with the gingival bleeding trait should be treated with caution, particularly when considering the relatively poor specificity seen in our own validation.

Another limitation of our periodontal information is the time difference between DNA extraction time and dental phenotype collection, and dental questionnaires which capture the history of periodontal conditions rather than current status. However, we excluded participants for whom the time difference between DNA extraction and dental questionnaires was over 5 years. We reasoned that subjects are likely to suffer from the same periodontal condition for roughly 5 years, because periodontal disease encompasses the nature of chronic inflammation.

Another limitation is our ability to address tissue specificity in disease-relevant tissues. Epigenetic changes are tissue-specific, and patterns in blood may not be shared across tissues. Tissues within oral cavity such as gingiva, buccal mucosa, and saliva are considered preferable to 
examine the regulatory genomic effects in periodontal disease due to their involvement or proximity to disease-affected areas [42]. Epigenetic modifications in the oral cavity are, to date, considered to take place mostly in epithelial tissue [43], and buccal swabs of the oral cavity typically contain the greatest amount of epithelial tissue [44]. We attempted to address this point by exploring methylation profiles in a modest sample of buccal tissue.

A further limitation was the lack of follow-up validation of the most associated signals in our study using a different DNA methylation profiling technology. For example, one of the peak signals in our results, cg21245277 in ZNF804A, contains several genetic variants in the probe sequence. Although these are not common genetic variants, they may still impact probe hybridization efficiency. It would therefore be valuable to validate the observed DNA methylation levels at this CpG site using a different technology, for example, bisulfite pyrosequencing. Furthermore, although the study subjects in our sample were representative of the general UK population and were not selected for major diseases, such as rheumatoid arthritis, the conclusions may not extend to all populations.

Although all of these drawbacks need to be further considered, our notable findings from the epigenome-wide analysis and candidate gene approach identify novel genomic regions in periodontitis and highlight candidate genes for further research. Previous studies examining epigenetic variation related to periodontitis have applied the case-control design with moderate samples and limited information on specific factors that may influence on DNA methylation such as age, gender, smoking status, and technical covariates. Overall, the current study suggests that epigenetic modifications may play an important role in the susceptibility and progression of periodontal disease. Future studies could focus on identifying environmental factors, which trigger DNA methylation changes relevant to periodontitis. For example, periodontal pathogenic bacteria could have the potential to cause alteration in host cellular DNA methylation status. Moreover, environmental factors such as aging, smoking, and stress can have impacts on epigenetic modifications. Further future work could explore in more depth the tissue specificity of epigenetic modifications in periodontitis. Tissue-shared epigenetic modifications are valuable as blood methylation data are available in other cohorts, which allow us to conduct replication studies. In the longer term, it may be feasible to use DNA methylation profiles towards personalized treatment and prevention of disease. For example, DNA methylation levels at the most associated signals in our study may be used to identify subjects at high risk of disease, towards modifications in exposures for specific environmental risk factors.

\section{Conclusions}

Epigenome-wide analyses and a candidate gene approach in adult female twins identified differentially methylated signals in periodontal disease. In conjunction with transcriptomics and metabolomics analyses, we conclude that the epigenetic changes may have functional regulatory effects over the course of development and progression of periodontal disease.

\section{Additional files}

Additional file 1: Table S1. Linear regression model describing summary statistics for the association between blood methylation at cg21245277 and gingival bleeding. (CSV 5 kb)

Additional file 2: Table S2. Linear regression model describing summary statistics for the association between blood methylation at cg08157914 and tooth mobility. (CSV 5 kb)

Additional file 3: Table S3. Linear regression model describing summary statistics for the association between blood methylation at cg11051055 and tooth mobility. (CSV 5 kb)

Additional file 4: Table S4. Covariate data and methylation beta values at cg21245277 in blood (CSV 91 kb)

Additional file 5: Table S5. Covariate data and methylation beta values at 58 tooth mobility significant CpG sites in blood. (CSV 410 kb)

Additional file 6: Table S6. Covariate data, gingival bleeding, and methylation beta values at 247 CpG-sites in blood. (CSV 1595 kb)

Additional file 7: Table S7. Covariate data, tooth mobility, and methylation beta values at 247 CpG sites in blood. (CSV 1486 kb)

Additional file 8: Table S8. Covariate data, tooth mobility, gingival bleeding, and methylation beta values at 248 CpG sites in buccal tissue for all subjects. (CSV $152 \mathrm{~kb}$ )

\section{Abbreviations}

BMIQ: Beta-mixture quantile normalization; DMPs: Differentially methylated positions; DZ: Dizygotic; EWAS: Epigenome-wide association scans; FDR: False Discovery Rate; GWAS: Genome-wide association scans; LMER: Linear mixedeffects regression; LPS: Lipopolysaccharide; MZ: Monozygotic; SNPS: Single nucleotide polymorphism

\section{Acknowledgements}

The authors would like to thank Dr. Tiphaine Martin for assistance with CoMET.

\section{Funding}

The study received support from the ESRC (ES/N000404/1 to J.T.B) and the JSPS (the Postdoctoral Fellowship for Research Abroad to Y.K.). The TwinsUK study was funded by the Wellcome Trust; European Community's Seventh Framework Programme (FP7/2007-2013); National Institute for Health Research (NIHR)- funded BioResource, Clinical Research Facility and Biomedical Research Centre based at Guy's and St Thomas' NHS Foundation Trust in partnership with King's College London. SNP genotyping was performed by The Wellcome Trust Sanger Institute and National Eye Institute via NIH/CIDR.

\section{Availability of data and materials}

Methylation profiles and covariates for candidate CpGs and CpGs significantly associated with periodontal traits are provided as supplementary data (Additional file 4: Table S4, Additional file 5: Table S5, Additional file 6: Table S6, Additional file 7: Table S7, Additional file 8: Table S8). The majority of the datasets analysed in the current study are available through GEO GSE62992 and GSE121633 (blood methylation), ArrayExpress E-MTAB-1866 (adipose methylation), and EGA EGAD00001001088 (blood expression). Additional individual-level data are not permitted to be shared or deposited due to the original consent given at the time of data collection. However, access to these data can be applied for through the TwinsUK data access committee. For information on access and how to apply http://twinsuk.ac.uk/resources-for-researchers/access-our-data/. 


\section{Authors' contributions}

YK and JTB designed the study and outlined the main conceptual ideas. JTB, TDS, and CJS generated the primary datasets, and $\mathrm{MI}$ and FH helped guide data generation and results interpretation. JTB, KSS, and CS oversaw the data analysis. YK led the data analysis. PC-T, JEC-F, ACA, JSE-SM, and CLR contributed to the data analysis. JTB and YK wrote the article, and all authors provided critical feedback and helped shape the research, analysis, and manuscript. All authors read and approved the final manuscript.

\section{Ethics approval and consent to participate}

Ethical approval was granted by the National Research Ethics Service London-Westminster, the St Thomas' Hospital Research Ethics Committee (EC04/015 and 07/H0802/84). All research participants have signed informed consent prior to taking part in any research activities.

\section{Consent for publication}

All authors have read and approved the manuscript for publication.

\section{Competing interests}

The authors declare that they have no competing interests.

\section{Publisher's Note}

Springer Nature remains neutral with regard to jurisdictional claims in published maps and institutional affiliations.

\section{Author details}

'Department of Twin Research and Genetic Epidemiology, School of Life Course Sciences, King's College London, London, UK. ²Department of Biomedical Sciences, Chang Gung University, Taoyuan, Taiwan. ${ }^{3}$ Division of Allergy, Asthma, and Rheumatology, Department of Pediatrics, Chang Gung Memorial Hospital, Linkou, Taiwan. ${ }^{4}$ Unit of Periodontology, Dental Institute, King's College London, London, UK.

\section{Received: 5 September 2018 Accepted: 11 January 2019} Published online: 13 February 2019

\section{References}

1. Rhodin K, et al. Chronic periodontitis genome-wide association studies: gene-centric and gene set enrichment analyses. J Dent Res. 2014;93(9): 882-90.

2. Offenbacher $\mathrm{S}$, et al. Genome-wide association study of biologically informed periodontal complex traits offers novel insights into the genetic basis of periodontal disease. Hum Mol Genet. 2016;25(10):2113-29.

3. Schroder NW, et al. Chronic periodontal disease is associated with singlenucleotide polymorphisms of the human TLR-4 gene. Genes Immun. 2005; 6(5):448-51.

4. de Brito Junior RB, et al. Polymorphisms in the vitamin $D$ receptor gene are associated with periodontal disease. J Periodontol. 2004;75(8):1090-5.

5. Kornman KS, et al. The interleukin-1 genotype as a severity factor in adult periodontal disease. J Clin Periodontol. 1997:24(1):72-7.

6. Shanmugam MK, Sethi G. Role of epigenetics in inflammation-associated diseases. Subcell Biochem. 2013:61:627-57.

7. Hirst M, Marra MA. Epigenetics and human disease. Int J Biochem Cell Biol 2009;41(1):136-46.

8. Oliveira NF, et al. DNA methylation status of the IL8 gene promoter in oral cells of smokers and non-smokers with chronic periodontitis. J Clin Periodontol. 2009;36(9):719-25.

9. Zhang $S$, et al. Interferon-gamma promoter hypomethylation and increased expression in chronic periodontitis. J Clin Periodontol. 2010; 37(11):953-61

10. Kobayashi T, Ishida K, Yoshie H. Increased expression of interleukin-6 (IL-6) gene transcript in relation to IL-6 promoter hypomethylation in gingival tissue from patients with chronic periodontitis. Arch Oral Biol. 2016;69:89-94.

11. Zhan $Y$, et al. Prioritization of candidate genes for periodontitis using multiple computational tools. J Periodontol. 2014;85(8):1059-69.

12. Schaefer AS, et al. A genome-wide association study identifies GLT6D1 as a susceptibility locus for periodontitis. Hum Mol Genet. 2010;19(3):553-62.

13. Laine ML, Crielaard W, Loos BG. Genetic susceptibility to periodontitis. Periodontol. 2012;58(1):37-68

14. Moayyeri $A$, et al. Cohort profile: TwinsUK and healthy ageing twin study. Int J Epidemiol. 2013;42(1):76-85.
15. Martin NG, Martin PG. The inheritance of scholastric abilities in a sample of twins. I. Ascertainments of the sample and diagnosis of zygosity. Ann Hum Genet. 1975;39(2):213-8.

16. Teschendorff $A E$, et al. A beta-mixture quantile normalization method for correcting probe design bias in Illumina Infinium 450 k DNA methylation data. Bioinformatics. 2013;29(2):189-96.

17. Consortium UK, et al. The UK10K project identifies rare variants in health and disease. Nature. 2015;526(7571):82-90.

18. Buil A, et al. Gene-gene and gene-environment interactions detected by transcriptome sequence analysis in twins. Nat Genet. 2015;47(1):88-91.

19. Harrow J, et al. GENCODE: the reference human genome annotation for The ENCODE Project. Genome Res. 2012;22(9):1760-74.

20. Li H, Durbin R. Fast and accurate short read alignment with BurrowsWheeler transform. Bioinformatics. 2009;25(14):1754-60.

21. Shin SY, et al. An atlas of genetic influences on human blood metabolites. Nat Genet. 2014;46(6):543-50.

22. Horvath S. DNA methylation age of human tissues and cell types. Genome Biol. 2013;14(10):R115

23. Brown, A.A., largeQvalue: a program for calculating FDR estimates with large datasets. bioRxiv, 2014

24. Neale MC, Cardon L, North Atlantic Treaty Organization, Scienti c Affairs Division. Methodology for genetic studies of twins and families. Dordrecht, Boston: Kluwer Academic Publishers; 1992.

25. Wickramasinghe $\mathrm{A}$, et al. Associations between the schizophrenia susceptibility gene ZNF804A and clinical outcomes in psychosis. Transl Psychiatry. 2015;5:e698.

26. Meadows JA, Wargo MJ. Carnitine in bacterial physiology and metabolism. Microbiology. 2015;161(6):1161-74.

27. Sakanaka A, et al. Arginine-ornithine antiporter ArcD controls arginine metabolism and interspecies biofilm development of Streptococcus gordonii. J Biol Chem. 2015;290(35):21185-98.

28. Kuboniwa $\mathrm{M}$, et al. Prediction of periodontal inflammation via metabolic profiling of saliva. J Dent Res. 2016:95(12):1381-6.

29. Devlin B, Roeder K. Genomic control for association studies. Biometrics. 1999:55(4):997-1004.

30. Reik W, Dean W, Walter J. Epigenetic reprogramming in mammalian development. Science. 2001:293(5532):1089-93.

31. Shang $D$, et al. Two-stage comprehensive evaluation of genetic susceptibility of common variants in FBXO38, AP3B2 and WHAMM to severe chronic periodontitis. Sci Rep. 2015;5:17882

32. Hayashi J, Masaka T, Ishikawa I. Increased levels of soluble CD14 in sera of periodontitis patients. Infect Immun. 1999;67(1):417-20.

33. Pietruska $\mathrm{M}$, et al. Evaluation of mCD14 expression on monocytes and the blood level of sCD14 in patients with generalized aggressive periodontitis. Adv Med Sci. 2006;51(Suppl 1):166-9.

34. Barnes VM, et al. Metabolomics reveals elevated macromolecular degradation in periodontal disease. J Dent Res. 2011;90(11):1293-7.

35. Riedel SL, et al. Lipid and fatty acid metabolism in Ralstonia eutropha: relevance for the biotechnological production of value-added products. Appl Microbiol Biotechnol. 2014;98(4):1469-83.

36. Choi YH, et al. Relationship between the burden of major periodontal bacteria and serum lipid profile in a cross-sectional Japanese study. Bmc Oral Health. 2018;18:77.

37. Kleber HP. Bacterial carnitine metabolism. FEMS Microbiol Lett. 1997;147(1):1-9.

38. Khongkhunthian S, et al. Raised chondroitin sulphate WF6 epitope levels in gingival crevicular fluid in chronic periodontitis. J Clin Periodontol. 2008; 35(10):871-6.

39. Kurushima $Y$ et al. Genetic and environmental contributions to the association between mood disorder and periodontal disease: A crosssectional study among female twins in the UK. J Clin Periodontol. 2019;46:40-50.

40. Eke PI, et al. Self-reported measures for surveillance of periodontitis. J Dent Res. 2013;92(11):1041-7.

41. Heaton B, et al. A clinical validation of self-reported periodontitis among participants in the Black Women's Health Study. J Periodontol. 2017;88(6):582-92.

42. Lokk K, et al. DNA methylome profiling of human tissues identifies global and tissue-specific methylation patterns. Genome Biol. 2014;15(4):r54.

43. Larsson L, Castilho RM, Giannobile W. Epigenetics and its role in periodontal diseases: a state-of-the-art review. J Periodontol. 2015:86(4):556-68.

44. Thiede $C$, et al. Buccal swabs but not mouthwash samples can be used to obtain pretransplant DNA fingerprints from recipients of allogeneic bone marrow transplants. Bone Marrow Transplant. 2000;25(5):575-7. 